International Journal on Artificial Intelligence Tools

(C) World Scientific Publishing Company

\title{
Interactive Learning of Pattern Rankings
}

\author{
Vladimir Dzyuba, Matthijs van Leeuwen, Siegfried Nijssen, Luc De Raedt \\ Department of Computer Science, KU Leuven \\ Celestijnenlaan 200A - bus 2402, Leuven, 3000, Belgium \\ \{vladimir.dzyuba, matthijs.vanleeuwen, siegfried.nijssen, luc.deraedt\}@cs.kuleuven.be
}

\author{
Received (Day Month Year) \\ Revised (Day Month Year) \\ Accepted (Day Month Year)
}

\begin{abstract}
Pattern mining provides useful tools for exploratory data analysis. Numerous efficient algorithms exist that are able to discover various types of patterns in large datasets. Unfortunately, the problem of identifying patterns that are genuinely interesting to a particular user remains challenging. Current approaches generally require considerable data mining expertise or effort from the data analyst, and hence cannot be used by typical domain experts.

To address this, we introduce a generic framework for interactive learning of userspecific pattern ranking functions. The user is only asked to rank small sets of patterns, while a ranking function is inferred from this feedback by preference learning techniques. Moreover, we propose a number of active learning heuristics to minimize the effort required from the user, while ensuring that accurate rankings are obtained. We show how the learned ranking functions can be used to mine new, more interesting patterns.

We demonstrate two concrete instances of our framework for two different pattern mining tasks, frequent itemset mining and subgroup discovery. We empirically evaluate the capacity of the algorithm to learn pattern rankings by emulating users. Experiments demonstrate that the system is able to learn accurate rankings, and that the active learning heuristics help reduce the required user effort. Furthermore, using the learned ranking functions as search heuristics allows discovering patterns of higher quality than those in the initial set. This shows that machine learning techniques in general, and active preference learning in particular, are promising building blocks for interactive data mining systems.
\end{abstract}

Keywords: Interactive data mining; preference learning; active learning; pattern mining.

\section{Introduction}

Large amounts of data are available nowadays. Making sense of this data and putting it to good use is a major challenge in many domains, ranging from academic research to practical commercial applications. However, the large sizes of datasets make manual processing and analysis virtually impossible. Automated tools that alleviate these issues are the subject of research in data mining and knowledge discovery.

*Also affiliated with Leiden Institute for Advanced Computer Science, Leiden University, The Netherlands. 
Pattern mining is an important concept in data mining that aims at discovering patterns from data. Informally, a pattern is an expression in a certain language that concisely describes some local structure in the dataset. Many variations of pattern mining have been proposed in the literature, and many algorithms for efficiently mining patterns from large datasets exist. For example, subgroup discovery ${ }^{29}$ concerns patterns in labeled data, i.e., descriptions of regions in the data characterized by an unusual distribution of the property of interest. In the context of a bank providing loans, the fact that $16 \%$ of loans with purpose = used car are not repaid may be an interesting subgroup pattern, in particular given the fact that the baseline of unpaid loans is only $5 \%$.

Unfortunately, the adoption of pattern mining techniques by domain experts is still limited in practice. One common problem is the so-called pattern explosion: large numbers of patterns are often discovered, of which many are very similar and hence redundant. Consequently, a domain expert has to invest substantial effort to identify those patterns that are relevant to her specific interests and goals. Manual filtering of the results or tuning algorithm parameters are hardly effective solutions and certainly hard for domain experts. Top-k mining and particularly pattern set mining ${ }^{28,33}$ are techniques that specifically address the redundancy problem; in both cases, the number of discovered patterns is limited.

Still, these existing approaches have a number of inherent problems, which correspond with the interestingness measure used. Objective interestingness measures, on one hand, only concern the structure of the data and do not take into account knowledge and goals of a user. As a result, re-discovery of common knowledge is a typical problem, even when redundancy is successfully avoided. Subjective interestingness measures, on the other hand, account for the user-specific context via a model of the dataset or of the entire domain. But then, an expert has to be familiar with the particular model type being used, e.g., Bayesian networks. This shifts the problem of the user from filtering results to specifying the right model, which is yet another non-trivial task and requires expertise beyond the problem domain.

\subsection{Learning task- and user-specific interestingness}

In practice, pattern interestingness heavily depends on the specific task and user characteristics, such as analysis goals and background knowledge. Developing principled methods to account for such information is key to broadening the adoption of pattern mining as a generic technique for knowledge discovery. Because of this strong dependency, we argue that direct involvement of the user in the mining process is essential. We propose to frame the problem as interactive learning and mining loop that consists of three major steps:

(1) Mining patterns.

In this step, a mining algorithm is used to find patterns that are to be presented to the user. It is crucial that the mining algorithm allows some form of subjective input. In general, this input is initially empty, and mining is essen- 
tially objective. However, in later iterations, an increasingly precise model of user interests becomes available, which allows for discovering subjectively more interesting patterns.

(2) Interacting with the user.

The patterns are presented to the user, who can freely explore and inspect them. Meanwhile, feedback is elicited from the user, either implicitly or explicitly. The feedback to the learning system should be simple in order for the system to be accessible to non-data mining experts, yet at the same time should convey enough information about the user's interests.

(3) Learning user-specific pattern interestingness.

The elicited feedback is used to build and improve the model of the user interests, i.e., to identify what makes patterns interesting to the user. Most importantly, the learned model is used in the next mining iteration, so that the effort required to achieve the analysis goals is minimized.

This generic loop can be paraphrased by the adage "Mine, interact, learn, repeat". Naturally, each step of the loop entails a number of important design choices. Questions that need to be answered include how to organize user interaction, how to model subjective interestingness, how to learn such models from user feedback, and how to incorporate subjective interestingness into pattern mining.

\subsection{Approach and contributions}

In this paper, we propose a concrete instance of the interactive learning and mining loop that is based on the notion of pattern rankings. For this, we build upon our recent work ${ }^{10}$, of which the current article is a substantially extended version. We will now outline our approach and contributions in more detail, and explicitly mention the novel contributions of this extended paper.

In our approach, which was initially introduced in our earlier work ${ }^{10}$, we model user-specific interestingness as a total order over patterns. That is, for any two patterns from a given pattern language, one of the two is deemed more interesting than the other. This results in a ranking over patterns, such that the most interesting patterns are ranked highest. It is such a pattern ranking that we would like to learn through interaction with the user.

To achieve this, we make the following assumptions about the user:

(1) A user has an implicit preference between any pair of patterns, which does not change during a particular analysis session;

(2) The costs of eliciting the complete preference relation, or pattern ranking, i.e., expressing it in any analytical or other form, are prohibitively high;

(3) For any single pair of patterns, however, the user can accurately identify which of the two she prefers, i.e., which pattern she considers more interesting.

Our first main contribution is a generic algorithm for the interactive learning of pattern rankings, which are represented by means of ranking functions. That is, we 
employ a preference learning algorithm to infer a ranking function that can score any pattern in the pattern language considered. The absolute scores provided by this function are unimportant, but the relative scores define the ranking over the complete pattern space. Ranking functions are functions over a feature representation of the patterns, so that feature relevance can be learned from user feedback.

Feedback elicited from the user amounts to rankings of small sets of patterns, to which we will refer as queries in this paper. Executing a query implies that the system selects a few patterns, presents these to the user, and asks the user to rank them. By generalizing from the pattern rankings provided by the user to such queries, a subjective pattern interestingness measure is learned. We propose and evaluate a number of active query selection methods, which aim to minimize the feedback -and thus effort- required from the user, while ensuring that accurate ranking functions are learned.

When mining the initial patterns, no knowledge about the user is known. Therefore, the user can select an objective interestingness measure based on her prior beliefs. This interestingness measure determines the initial source ranking; the closer this ranking is to the subjective target ranking that is to be learned, the easier the learning task becomes. When a pool of patterns has been mined, queries can be selected and presented to the user. The ranking function is then updated based on feedback provided by the user, and then the learned ranking function is used to mine novel, hopefully more interesting patterns.

The second main contribution is the application of the proposed approach in the context of two well-known pattern mining settings, i.e., frequent itemset mining and subgroup discovery. The former concerns the discovery of items that frequently cooccur in unlabeled, transactional data, whereas the latter concerns the discovery of subsets of labeled data for which the labels deviate from the overall distribution. Our previous work only considered subgroup discovery, hence the application to frequent itemset mining is a novel contribution of this paper.

The remainder of this paper is organized as follows. First, Sections 2 and 3 describe related work and preliminaries respectively. Then, Section 4 describes a toy example to illustrate how our framework interactively learns pattern rankings. Section 5 introduces our framework for learning pattern rankings, consisting of both a problem definition and detailed algorithm description, after which Section 6 discusses how to use the learned ranking functions for mining new patterns.

Section 5 presents the extensive (and extended) experimental evaluation, for both frequent itemset mining and subgroup discovery. In order to perform a principled and objective evaluation of our methods, we emulate user preferences over patterns using several existing interestingness measures. The results show that the algorithm is able to learn accurate pattern rankings, and that query selection heuristics help reduce the amount of input required for learning. Moreover, the learned ranking functions generalize well and allow discovering novel high-quality patterns when used for mining. After that, we round up with a discussion and conclusions in Sections 8 and 9. 


\section{Related work}

In this section we describe work that is most closely related to ours, on the topic of subjective interestingness and interactive pattern mining on one hand, and on the topic of preference learning on the other hand.

Subjective interestingness and interactive pattern mining The importance of taking user knowledge and goals into account in order to discover genuinely interesting patterns was first emphasized by Tuzhilin ${ }^{25}$ in 1995. Nevertheless, most works concerning user-specific pattern interestingness measures and interactive pattern mining are fairly recent ${ }^{26}$. These approaches can be divided into three groups: 1) specifying a model of user interests in advance; 2) using user feedback to directly influence the search procedure; and 3) learning an explicit model of user interests.

Modeling user interests The approach by Jaroszewicz et al. ${ }^{13}$ allows a user to specify a Bayesian network that represents beliefs about the data-generating process. The algorithm then finds surprising attribute sets, i.e., attribute sets for which the discrepancy between the expected and observed frequencies is larger than a certain threshold. The search is based on efficiently computing (or approximating) a large number of marginal distributions. A user can then manually update the Bayesian network, i.e., her beliefs, based on the inspected patterns, and subsequently repeat the mining process. One disadvantage of this approach is that it does not avoid the pattern explosion, at least not without tuning a threshold.

De $\mathrm{Bie}^{8}$ has developed a general framework for exploratory data analysis that uses information theory to formalize subjective interestingness as surprisingness with respect to certain prior beliefs. Different types of prior beliefs can be used, for example, expected frequencies of individual items. Given these prior beliefs, a Maximum Entropy distribution is fit to represent the expected data. This distribution is then used to quantify how informative the pattern is, given the beliefs. The framework lends itself well to iterative data mining: starting from a model based solely on prior beliefs, one can look for the subjectively most interesting pattern, which can then be added to the model. Hence, the next discovered pattern will automatically be substantially different from the previous one; this helps avoiding redundancy. A disadvantage of this framework is that it currently only allows for scoring pre-mined pattern collections, but not for directly mining high-scoring patterns.

Interactive search Bhuiyan et al. $^{3}$ proposed a technique that is based on Markov Chain Monte Carlo sampling of frequent itemsets. User interests are modeled via a scoring function that is a product of weights of individual items; the probability of sampling an itemset is proportional to its score. In each iteration, a user inspects a small set of sampled itemsets and provides feedback by liking or disliking them. This feedback is then used to update the weights: the weights of items comprising liked (resp. disliked) itemsets are increased (resp. decreased). 
As a result, itemsets that are more similar to liked ones become more likely to be sampled and presented to the user. Sampling itemsets allows avoiding pattern explosion, while still ensuring obtaining a sample of the complete set of patterns that is representative according to the (current) sampling distribution.

Dzyuba and Van Leeuwen ${ }^{9}$ developed a similar approach for interactive subgroup discovery. They augment a beam search-based subgroup discovery algorithm DSSD with interactive beam selection: at each level, a user is allowed to inspect subgroups in the current beam and like or dislike them. This feedback is used to prune the beam and to re-weigh an objective subgroup quality measure, which essentially becomes subjective. The goal is to steer the search towards patterns that are more similar to the liked ones. Even though the algorithm uses a naive scheme to accomplish this, which does not always have predictable outcomes, it has been shown to improve the results from the subjective standpoint of a domain expert.

Learning models of user interests Preference learning has been previously used to identify interesting patterns in an interactive manner. Xin et al. ${ }^{30}$ investigated learning a user-specific ranking of frequent patterns (primarily itemsets and sequences). A clustering-based method similar to information retrieval approaches is used to select patterns for feedback. However, they only consider a specific learning target based on the discrepancy between the expected and observed supports of a pattern, and they do not use the learned functions to search for novel patterns.

Rueping ${ }^{22}$ demonstrated the feasibility of learning subgroup rankings and applying learned ranking functions to discover high-quality subgroups. However, Rueping does not discuss active learning aspects and uses a custom variant of the learner and data modifications that are specific to subgroup discovery. Therefore, it cannot be straightforwardly generalized to other pattern mining tasks, as we do in this paper.

The One Click Mining system ${ }^{4}$ is a generic system for interactive data mining that is not restricted to a single pattern type. That is, contrary to our system, it considers multiple types of patterns at once. Essentially, it learns two types of preferences simultaneously. On one hand, it uses a multi-armed bandit strategy to learn which mining algorithms discover the patterns that are most positively evaluated by a user. For example, that the user prefers subgroups with a particular target to itemsets. These preferences are used to allocate computation time to the different algorithms. On the other hand, co-active learning is used to learn a pattern ranking function from implicit user feedback, i.e., the actions performed by the user in the graphical user interface. Conceptually, this system is very similar to the one proposed in this paper, using similar techniques. Still, there are a number of key differences. One such difference is that One Click Mining only mines objectively interesting patterns, i.e., the learned ranking function is not used in the mining phase. Another difference is that although we show that our framework is generic enough to deal with different types of patterns, we choose to focus on one mining task at a time, mainly for reasons of transparency and ease of use for the user. 
Recently, Negrevergne et al. ${ }^{19}$ proposed dominance programming, a declarative language that allows one to explicitly specify pairwise preferences between patterns, which are then used to find a complete set of non-dominated patterns. Only modeling objective preferences has been studied so far; learning models from example preferences is an open research question.

Preference learning In this paper we use preference learning ${ }^{12}$, a research area encompassing several tasks related to learning preferences within the field of machine learning. In particular, we deal with an instance of the object ranking problem, i.e., acquiring ranking functions from sample orders ${ }^{15}$.

Active object ranking is related to the problem of learning to rank in information retrieval, as both aim at learning a ranking from a minimum number of sample rankings. A number of general heuristics aimed at improving top results of search engines were developed ${ }^{24,32}$. Methods that specifically target object ranking algorithms exploit probabilistic models of a document collection ${ }^{21}$ or relations between documents ${ }^{31}$. A theoretical analysis of query complexity of active object ranking has been presented recently ${ }^{2}$.

\section{Preliminaries}

This section provides preliminaries on pattern mining, with a focus on the two well-known instances that we consider in this paper, frequent itemset mining ${ }^{1}$ and subgroup discovery ${ }^{16}$.

Pattern mining aims to reveal structure in data in the form of patterns, with a strong emphasis on obtaining comprehensible descriptions. Formally, the pattern mining task is defined as follows ${ }^{18}$. Given a dataset $\mathcal{D}$, a language $\mathcal{L}$ defining subsets of $\mathcal{D}$ (for example, logical formulae over domains of attributes), and a selection predicate $q$ that determines whether an element $p \in \mathcal{L}$ describes an interesting subset of $\mathcal{D}$, the task is to find descriptions of all interesting subsets, i.e., $\{p \in \mathcal{L} \mid q(p, \mathcal{D})$ is true $\}$. Therefore, a pattern consists of a description $p$ and a corresponding cover $C_{p}=\{T \in \mathcal{D} \mid p(T)$ is true $\}$, i.e., the subset of $\mathcal{D}$ covered by this description (we omit the subscript $p$ whenever it is clear from the context).

A dataset $\mathcal{D}$ is typically a bag of tuples over a certain set of attributes $\mathcal{A}$. Let $\mathcal{A}=\left\{A_{1}, \ldots, A_{l-1}, A_{l}\right\}$ denote a set of attributes, where each attribute $A_{j}$ has a domain of possible values $\operatorname{Dom}\left(A_{j}\right)$. Then, a dataset $\mathcal{D}=\left\{T_{1}, \ldots, T_{n}\right\} \subseteq$ $\operatorname{Dom}\left(A_{1}\right) \times \ldots \times \operatorname{Dom}\left(A_{l}\right)$ is a bag of tuples over $\mathcal{A}$.

In most cases, the selection predicate $q$ includes a constraint on the minimal size of the cover $C$, or minimal frequency of a pattern in $\mathcal{D}$, i.e., $\{p \in \mathcal{L} \mid$ Frequency $(p) \geq \sigma\}$, where Frequency $(p)=\frac{\left|C_{p}\right|}{|\mathcal{D}|}$ and $\sigma$ is a user-provided frequency threshold. Such settings are referred to as frequent pattern mining. Top$k$ mining is an alternative setting where the task is to find the $k$ highest ranked patterns with respect to a given interestingness measure $\varphi$, where $\mathrm{k}$ is the desired number of solutions.. 
Itemset mining is concerned with finding patterns in binary data, i.e., $\forall j \operatorname{Dom}\left(A_{j}\right)=\{0,1\}$. Attributes are traditionally called items and the complete set of items is denoted by $\mathcal{I}$. Then, tuples in $\mathcal{D}$ are subsets of $\mathcal{I}$, i.e., $T_{i} \subseteq \mathcal{I}$. The pattern language also consists of subsets of $\mathcal{I}$, i.e., $\mathcal{L}=2^{\mathcal{I}}$. A tuple $T$ is covered by an itemset $I \in \mathcal{L}$ iff $I \subseteq T$. Frequent itemset mining is a well-studied instance of itemset mining. However, due to the pattern explosion, alternative formulations have been proposed, e.g., top- $k$ mining of surprising itemsets, where Surprisingness $(I, \mathcal{D})=$ Frequency $(I)-\prod_{i \in I}$ Frequency $(\{i\})$.

Subgroup discovery, an instance of supervised descriptive rule discovery ${ }^{17}$, is concerned with finding subsets of a dataset that have a substantial deviation in a property of interest, compared to the entire dataset. This implies that an attribute $A_{l}$ is considered the target attribute, whereas other attributes are description attributes. In this paper we only consider a single binary target attribute, but generalizations are possible. There are no constraints on the domains of the description attributes. The pattern language consists of conjunctions of conditions over description attributes, e.g., $A_{1}=a \wedge A_{2}>0$. Subgroup discovery is typically formalized as a top- $k$ mining problem. Let $C^{l}$ (resp. $\mathcal{D}^{l}$ ) denote the set of tuples with label $l$ in the subgroup cover (resp. in the entire dataset). Examples of subgroup quality measures include Sensitivity $(p)=\frac{\left|C^{1}\right|}{\left|\mathcal{D}^{1}\right|}$, Specificity $(p)=1-\frac{\left|C^{0}\right|}{\left|\mathcal{D}^{0}\right|}$, and

$$
\chi^{2}(p)=\sum_{l \in\{0,1\}} \frac{\left(|C|\left(\left|C^{l}\right|-\left|\mathcal{D}^{l}\right|\right)\right)^{2}}{|C|\left|\mathcal{D}^{l}\right|}+\frac{\left(|C|\left(\left|C^{l}\right|-\left|\mathcal{D}^{l}\right|\right)\right)^{2}}{(|\mathcal{D}|-|C|)\left|\mathcal{D}^{l}\right|}
$$

For example, the dataset credit-g (see Section 7) contains information about 1000 loans. The target attribute indicates whether a loan has been repaid (positive label) or not (negative). The entire dataset contains 700 positive tuples. The subgroup with description checking_status $=$ no_checking covers 394 instances, out of which 348 are positive. It has the highest value of $\chi^{2}$ among all subgroups with one condition in the description: $\chi^{2}(p)=103.96$.

\section{Interactive learning - an example}

The following toy example illustrates how the proposed approach can be applied in a data analysis session. Let us assume a subgroup discovery setting and a dataset $\mathcal{D}$ defined over three binary attributes $\mathcal{A}=\left\{A_{1}, A_{2}, A_{T}\right\}$, where $A_{T}$ is the target attribute (see Table 1). Furthermore, assume that a user is interested in the relation be-

Table 1. A toy dataset

\begin{tabular}{rrr|r}
\hline & $A_{1}$ & $A_{2}$ & $A_{T}$ \\
\hline$T_{1}$ & 1 & 1 & 1 \\
$T_{2}$ & 1 & 0 & 1 \\
$T_{3}$ & 0 & 1 & 0 \\
\hline
\end{tabular}
tween $A_{2}$ and $A_{T}=0$, e.g., that a certain property of a loan makes it risky, unless it has other properties. Note that this does not imply that the user knows this a priori, but rather that she would find this pattern interesting once it is shown to her. Hence, asking the user to express this in advance of mining is complicated, but we can hopefully learn this. 
For this small toy example, it is feasible to mine and present all subgroups that occur in the data; see Table 2. Moreover, given the above assumption on user interest, we can define a desired, subjective 'target ranking' according to which the patterns should be ranked. That is, there is a ground truth that can be used to emulate user feedback and that we would like to learn. The subgroups in Table 2 are ranked according to this subjective target ranking (leftmost column).

Table 2. All subgroups that occur in the toy dataset of Table 1, ranked according to the desired subjective ranking. For each subgroup, its description is given, together with absolute frequencies (all, positive, and negative tuples respectively), sensitivity and specificity.

\begin{tabular}{rl|rrrrr}
\hline Rank & Description $p$ & $|C|$ & $\left|C^{+}\right|$ & $\left|C^{-}\right|$ & Sensitivity & Specificity \\
\hline 1 & $A_{1}=0 \wedge A_{2}=1$ & 1 & 0 & 1 & 0 & 0 \\
2 & $A_{2}=1$ & 2 & 1 & 1 & 0.5 & 0 \\
3 & $A_{2}=0$ & 1 & 1 & 0 & 0.5 & 1 \\
4 & $A_{1}=1 \wedge A_{2}=0$ & 1 & 1 & 1 & 0.5 & 0 \\
5 & $A_{1}=0$ & 1 & 0 & 1 & 0 & 0 \\
6 & $A_{1}=1$ & 2 & 2 & 0 & 1 & 1 \\
7 & $A_{1}=1 \wedge A_{2}=1$ & 1 & 1 & 1 & 0.5 & 0 \\
\hline
\end{tabular}

In practice, a user often performs top- $k$ mining with respect to an objective quality measure, and with additional constraints to restrict the results. Here, we assume that the user decides to mine all subgroups consisting of a single condition and rank them according to Specificity. The resulting subgroups and ranking is shown in Table 3. The obtained ranking does clearly not match our user's desired, subjective ranking. Without our framework, this would be the end result and the user would either have to be happy with the results, or tune the algorithm parameters based on these results. The former is unsatisfactory, while the latter is hard: what other interestingness measure and parameters should we use to obtain more interesting patterns?

Table 3. Initial subgroups obtained by mining top patterns w.r.t. Specificity, limited to patterns consisting of a single condition.

\begin{tabular}{lrr}
\hline & Specificity & Subjective rank \\
\hline$A_{1}=1$ & 1 & 6 \\
$A_{2}=0$ & 1 & 3 \\
$A_{1}=0$ & 0 & 5 \\
$A_{2}=1$ & 0 & 2 \\
\hline
\end{tabular}

The goal of our interactive pattern mining framework is to assist the user in data exploration. To this end, the algorithm proposes the user to inspect and compare small sets of subgroups. In this example, let us assume that the subgroups $\left\{A_{1}=1 ; A_{2}=0 ; A_{1}=0\right\}$ are selected and shown to the user. The system should 
provide the user with the tools necessary to inspect and understand the patterns (for example, by means of data visualization); these issues are actively researched in the fields of human-computer interaction and visual analytics.

With the help of these tools, the user gains an understanding of the current patterns, the data, and of her interests, which enables her to provide feedback. In this case, the ranking $A_{2}=0 \succ A_{1}=0 \succ A_{1}=1$ would be given, as this coincides with the subjective, implicit ranking we assume the user to have. This implies that $A_{2}=0$ is deemed subjectively most interesting, while $A_{1}=1$ is the least interesting of the three.

Next, the algorithm uses the obtained feedback to learn a (subjective) pattern ranking function. To be able to use existing learning algorithms for this purpose, patterns are represented as numeric feature vectors (the details are explained in Section 6). Then, RANKING SVM is applied to learn a linear ranking function $h(\vec{p})=\vec{w} \cdot \vec{p}$, i.e., a vector of pattern feature weights defining a ranking function that is consistent with the user feedback.

To improve the ranking function, the interaction and learning steps can be repeated a number of times. In our example, the algorithm queries another set of subgroups $\left\{A_{2}=0 ; A_{2}=1 ; A_{1}=1\right\}$, obtains the ranking $A_{2}=1 \succ A_{2}=0 \succ A_{1}=1$ as feedback, and learns a new weight vector for $h$. The effect of interaction and learning is shown in Table 4. After two iterations, the learned ranking is almost identical to the desired target ranking, and certainly much better than the initial ranking based on specificity. This is also reflected by Spearman's rank correlation coefficients between the obtained and target rankings, indicated by $\rho$.

This concludes one full iteration of the mine, interact, and learn loop, after which the whole procedure can be repeated. The second loop is executed as the first, except that the learned ranking function is now used to mine and rank patterns.

For this toy example, we evaluate the generalization capacity of the learned ranking function by applying it to the complete set of subgroups from Table 2 . The results are presented in Table 5 . We observe that the learned ranking function generalizes very well: when the subgroups are sorted according to this function, the resulting ranks are very close to those of the desired target ranking. In other words, the learned ranking is highly correlated with the target ranking, indicated

Table 4. Learning a desired target ranking from user input. Each iteration of feedback and learning improves the approximation of the target ranking. $h$ denotes the absolute score assigned by the ranking function, $\rho$ is the Spearman's rank correlation coefficient between the indicated and target rankings.

\begin{tabular}{l|lr|lr|l}
\hline Initial & After iteration 1 & $h$ & After iteration 2 & $h$ & Target \\
\hline$A_{1}=1$ & $A_{2}=0$ & -0.01 & $A_{2}=1$ & -0.01 & $A_{2}=1$ \\
$A_{2}=0$ & $A_{1}=0$ & -0.02 & $A_{1}=0$ & -0.01 & $A_{2}=0$ \\
$A_{1}=0$ & $A_{2}=1$ & -0.03 & $A_{2}=0$ & -0.02 & $A_{1}=0$ \\
$A_{2}=1$ & $A_{1}=1$ & -0.06 & $A_{1}=1$ & -0.1 & $A_{1}=1$ \\
\hline$\rho=-0.8$ & $\rho=0.4$ & $\rho=0.8$ & \\
\hline
\end{tabular}


Table 5. Generalization capacity of the learned ranking function. All subgroups from Table 2 are ranked according to $h$.

\begin{tabular}{lrrr}
\hline Description & $h$ & Learned rank & Target rank \\
\hline$A 1=0 \wedge A 2=1$ & 0.01 & 1 & 1 \\
$A 2=1$ & -0.01 & 2 & 2 \\
$A 1=0$ & -0.01 & 3 & 5 \\
$A 2=0$ & -0.02 & 4 & 3 \\
$A 1=1 \wedge A 2=1$ & -0.04 & 5 & 7 \\
$A 1=1 \wedge A 2=0$ & -0.04 & 6 & 4 \\
$A 1=1$ & -0.1 & 7 & 6 \\
\hline
\end{tabular}

by a high correlation coefficient of $\rho=0.8$. This implies that if $h$ were used as interestingness measure in top- $k$ mining, subjectively more interesting subgroups would be discovered.

In the following sections, we formalize the problem and describe the algorithms required to implement the proposed workflow, i.e., we discuss techniques for learning rankings, query selection methods, and feature representations for patterns.

\section{Interactive learning of pattern rankings}

We now introduce the formal definition of the pattern ranking learning task as well as algorithms for solving this task.

\subsection{Learning pattern rankings}

The pattern ranking task is formally defined as follows. Recall that $\mathcal{L}$ denotes the pattern language, that is, the universal set of all possible patterns. We will assume that there is an unknown, user-specific target ranking $R^{*}$, that is, a total order over $\mathcal{L}$. We shall write $p \succ q$ when $p$ is preferred over $q$ according to $R^{*}$. The goal of learning will be to learn an approximation $\hat{R}$ of $R^{*}$ on the basis of the feedback provided by the user. We make the following assumptions:

The feedback takes the form of example rankings $f=p_{f_{1}} \succ \ldots \succ p_{f_{n}}$; these are total strict orders over subsets of $\mathcal{L}$. Feedback will be obtained through interaction with the user.

Each hypothesis $h$ (in the hypothesis space $\mathcal{H}$ ) is a ranking function $h$ that maps descriptions of patterns to real values and defines a ranking as follows: $p_{i} \succ_{h} p_{j}$ if and only if $h\left(p_{i}\right)>h\left(p_{j}\right)$.

Each pattern $p \in \mathcal{L}$ will be represented by a feature vector $\vec{p}=\left[x_{1}, \ldots, x_{m}\right]$.

The goal is to learn an approximation $\hat{R}$ of the target ranking $R^{*}$ that minimizes the loss function, on the basis of a set of examples $F$ (a set of example rankings) provided by the user. Note that there is not necessarily a hypothesis $h^{*} \in \mathcal{H}$ that correctly represents the unknown target ranking $R^{*}$. This depends both on the hypothesis space $\mathcal{H}$ considered and the specific target ranking. 


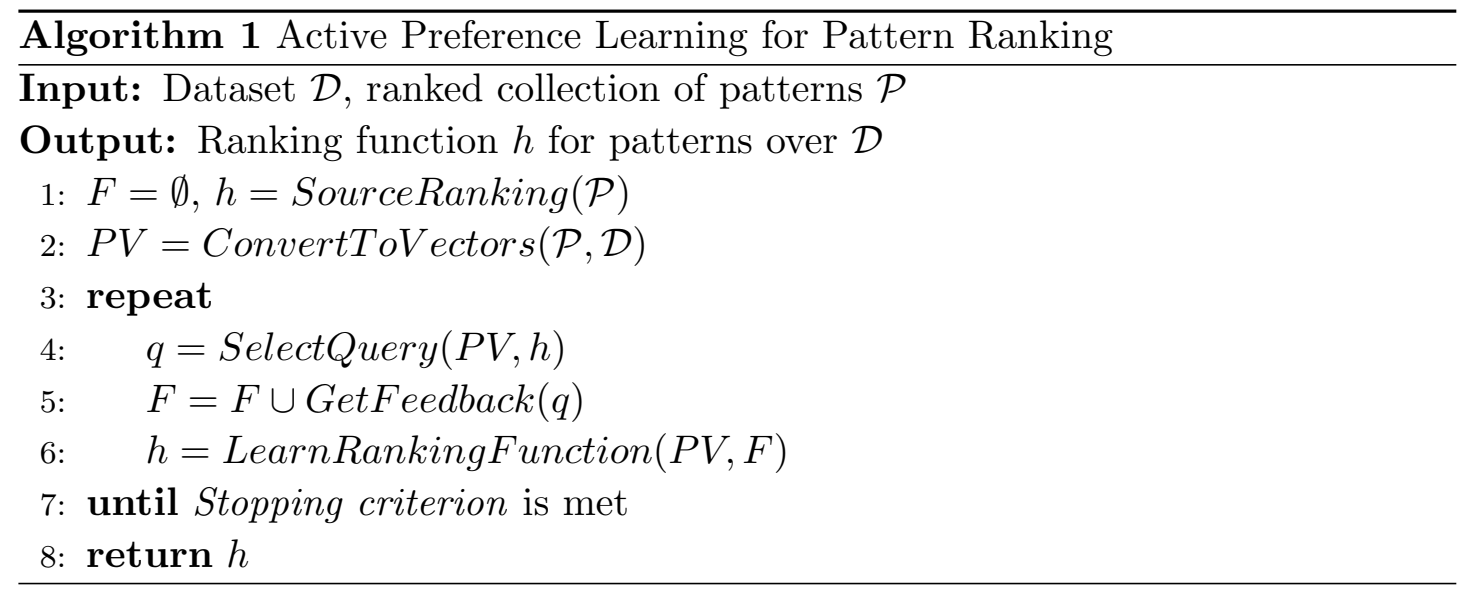

The pattern ranking task as just defined can be seen as an instance of object ranking, for which various types of loss functions and learning algorithms have been described. Two principal categories of object ranking techniques are pairwise and listwise methods.

Pairwise algorithms treat each sample ranking as a set of corresponding ranked pairs. For example, $\vec{p}_{1} \succ \vec{p}_{2} \succ \vec{p}_{3}$ corresponds to $\left\{\left(\vec{p}_{1} \succ \vec{p}_{2}\right),\left(\vec{p}_{1} \succ \vec{p}_{3}\right),\left(\vec{p}_{2} \succ \vec{p}_{3}\right)\right\}$. In the pairwise setting, the loss that needs to be minimized is a function of the number of incorrectly ranked pairs in the training data:

$$
\operatorname{Loss}_{\text {pairwise }}(\hat{R}, F)=\sum_{f_{k} \in F} \sum_{\left(\vec{p}_{i k} \succ \vec{p}_{j k}\right) \in f_{i}} L\left(\hat{R}, \vec{p}_{i k}, \vec{p}_{j k}\right)
$$

Listwise algorithms do not reduce the rankings to pairs, i.e., each sample ranking constitutes one training example. Hence, the loss function is defined over complete rankings:

$$
\operatorname{Loss}_{\text {listwise }}(\hat{R}, F)=\sum_{f_{k} \in F} L\left(\hat{R}, f_{k}\right)
$$

The pattern ranking task can be solved by finding a ranking $\hat{R}$ that minimizes either a pairwise or a listwise loss function. Since we will use and evaluate instances of both, we will not state a preference here.

\subsection{Algorithm for learning pattern rankings}

We now present a generic algorithm for learning pattern ranking functions. Algorithm 1 receives a collection of patterns $\mathcal{P} \subset \mathcal{L}$ as input. The initial pattern collection can be mined using any standard pattern mining algorithm, and is ranked according to an objective interestingness measure. This initial ranking is referred to as the source ranking.

In order to apply preference learning, patterns are represented as vectors of numeric features (Line 2); this will be discussed in detail in Section 6 .

Within the interaction and learning loop, query selection methods select sets of patterns that will be shown to the user (Line 4). Assuming that the query size is 
fixed, the goal is to minimize the number of queries required to attain a certain ranking accuracy. The methods take into account factors such as the current estimated interestingness of a pattern, the estimation uncertainty, the diversity of the query, and/or the structure of the data.

A user provides feedback to the queries in the form of rankings (Line 5). This feedback format is computationally more expensive for a user than graded feedback, i.e., assigning scores from a predefined scale. However, we argue that it has two advantages. First, it requires neither a deep understanding of the scale by a user, nor a thorough scale calibration. Second, graded feedback can be converted to the ordered format, albeit at a cost of reduced granularity.

The ranked queries are then used as training data for an object ranking algorithm. When the pairwise loss function is used, the problem is similar to classification. In fact, many pairwise algorithms are extensions of classification algorithms, e.g., Ranking SVM ${ }^{14}$, RankBoost ${ }^{11}$, or RAnkNeT ${ }^{6}$. Moreover, Stochastic Coordinate Descent (SCD) for logistic-loss ${ }^{23}$ can be easily adapted to perform pairwise preference learning.

Listwise algorithms are designed specifically for the object ranking task. For example, LISTNET ${ }^{7}$ uses neural networks and gradient descent to minimize the loss function based on the probabilistic model of permutations. We evaluate the performance of various object ranking algorithms for pattern ranking in Section 7 .

The interaction and learning loop stops when a certain stopping criterion is met (Line 7). Such criteria can consider marginal effects of additional queries on the learned ranking or limit the maximal user effort. Alternatively, the user can manually stop the algorithm, as soon as she considers her information need satisfied. In the experiments, we stop the learning after a fixed number of iterations.

\subsection{Active learning techniques}

Active preference learning is a challenging problem. Selecting an optimal query is NP-hard ${ }^{2}$, therefore in most cases exact query selection methods are computationally too expensive to be used in interactive settings. Consequently, heuristic methods are commonly used.

Query selection methods balance exploration of the pattern space with exploitation of available preference feedback. In the context of pattern mining, the source ranking is a strong starting point. The common method to ensure sufficient exploration is to maintain diversity among queried objects. We consider two categories of heuristics: greedy heuristics inspired by methods from information retrieval (IR), which explicitly take objective quality measures into account, and uncertainty-based heuristics specific to the RANKING SVM learner.

IR-inspired heuristics IR-inspired heuristics were initially developed in the context of improving search engines, hence they inherently aim at identifying a small number of top-ranking objects (documents). These greedy heuristics rely on the 
availability of an objective quality measure (relevance). The query selection process always starts from a set including the currently top-ranked pattern and proceeds with greedily selecting patterns that maximize the heuristic. Let $p$ denote a candidate pattern, and $q$ the current (incomplete) query.

When applying these heuristics, we start from the raw values of the source pattern interestingness measure $\varphi$, and progressively interpolate the values of the learned ranking function in order to take into account the current estimation of the target ranking:

$$
\operatorname{Quality}(p)=\mu h(p)+(1-\mu) \varphi(p),
$$

where $\mu$ is an interpolation parameter and $h$ is the learned ranking function.

MMR (Maximal Marginal Relevance) ${ }^{24}$ aims to select a high-quality pattern that is dissimilar from already selected patterns. Dissimilarity is defined as the minimal distance to an already selected pattern, e.g., the Euclidean distance between pattern vectors. The parameter $\alpha \in[0 ; 1]$ is a quality-diversity trade-off parameter.

$$
\begin{array}{r}
M M R(p, q)=\alpha \operatorname{Quality}(p)+(1-\alpha) \operatorname{Diversity}(p, q) \\
\text { where Diversity }(p, q)=\min _{p^{\prime} \in q} \operatorname{dist}\left(p, p^{\prime}\right)
\end{array}
$$

RDD (Relevance, Diversity, and Density) ${ }^{32}$ exploits the structure in $\mathcal{P}$ by adding a density term. The intuition behind this approach is that querying patterns from dense regions provides more information about preferences. Density of a region around a pattern is quantified as the average distance to all other patterns.

$$
\begin{array}{r}
R D D(p, q)=\alpha \operatorname{Quality}(p)+\beta \operatorname{Density}(p, \mathcal{P})+ \\
+(1-\alpha-\beta) \operatorname{Diversity}(p, q) \\
\text { where Density }(p, \mathcal{P})=\frac{1}{|\mathcal{P}|} \sum_{p^{\prime} \in \mathcal{P}} \operatorname{dist}\left(p, p^{\prime}\right)
\end{array}
$$

MMR and RDD only maintain local diversity, i.e., diversity within the current query. We aim to exploit global diversity, i.e., diversity between the queries, by introducing a new heuristic GLOBALMMR. It is an extension of MMR, where the diversity term is redefined as $\operatorname{Diver} \operatorname{sity}(p, Q)=\min _{p^{\prime} \in Q} \operatorname{dist}\left(p, p^{\prime}\right)$, where $Q=$ $q \cup \bigcup_{f_{i} \in F} f_{i}$ is the union of all queries, including the current incomplete one.

In all computations, values of the quality measure, the learned ranking function, and the distance measure are normalized to the range $[0 ; 1]$. For the quality measure and the learned ranking function, the minimal and the maximal values over $\mathcal{P}$ are used as range limits. For distance, the upper limit is estimated by the diameter of the object set, i.e., $\max _{p_{i}, p_{j} \in \mathcal{P}} \operatorname{dist}\left(p_{i}, p_{j}\right)$.

Uncertainty-based heuristics SVMBATCH, presented in Algorithm 2, is a straightforward extension of the batch query selection method for classification SVMs by Brinker ${ }^{5}$. This method aims at selecting a diverse set of examples with 


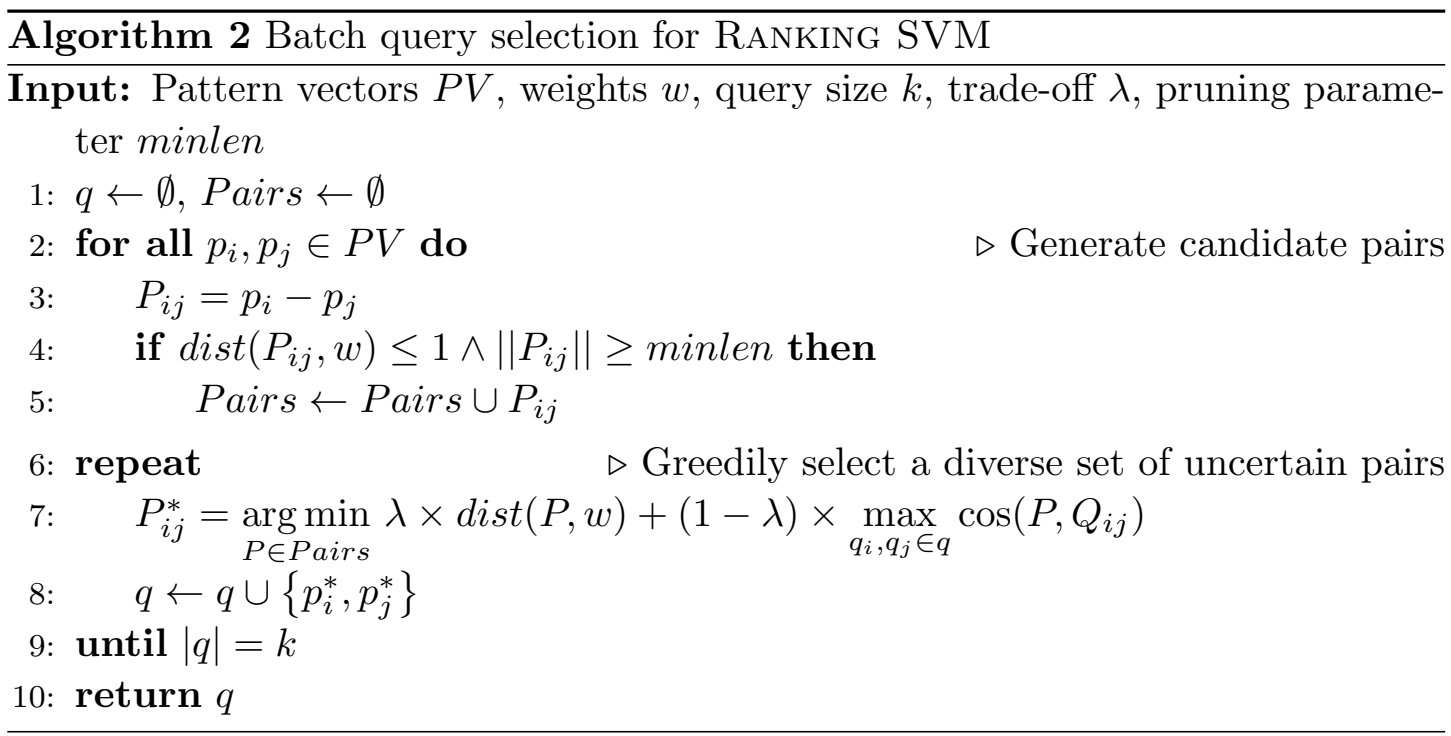

high prediction uncertainty. Uncertainty is quantified as the distance of a candidate example to the margin, whereas diversity is quantified by maximal cosine similarity between an example and already selected examples. This method only considers examples that lie on or within the margins.

In case of pairwise preferences, an individual example is a pair of patterns. Pairs are explicitly represented as differences between respective pattern vectors, similar to their representation in the RANKING SVM formulation. The total number of candidates is proportional to $|\mathcal{P}|^{2}$, therefore in order to reduce computational costs we introduce an additional pruning step. All pair vectors $P_{i j}$ for which $\left\|P_{i j}\right\|<$ minlen are removed from the candidate set. The intuition behind this pruning technique is that pair vectors with low norms correspond to highly similar patterns, and reducing uncertainty of predicting relative positions of similar patterns is less useful for learning a general ranking. Note that the distance between a pair vector $P_{i j}$ and the hyperplane is proportional to the difference between values of the ranking function for $p_{i}$ and $p_{j}$. However, the exact value has to be computed explicitly. Recently, Qian et al. ${ }^{20}$ proposed a similar active learning heuristic targeted at RANKING SVM, where efficiency is ensured by combining locality-sensitive and uncertainty hashing.

Preliminary experiments confirmed the utility of batch querying, e.g., querying the union of the two most informative pairs yields a larger performance improvement than three consecutive queries of the single most informative pair (in both cases 6 pairs are queried). Pruning reduces the runtime and can have a positive impact on learning performance; see Section 7 for experiments.

\subsection{Mining using learned ranking functions}

As also demonstrated in the toy example in Section 4, the learned ranking function generalizes beyond the training data $F$ and the input pattern set $\mathcal{P}$. Hence, it can 
be regarded as a general subjective interestingness measure defined over the pattern language $\mathcal{L}$, for the current user and dataset $\mathcal{D}$. It can be used to discover novel patterns that are likely to be interesting to the user.

A straightforward approach to accomplish this is to use the ranking function $h$ directly as a search heuristic. In addition to $h$, a search algorithm needs access to the function that converts a pattern to its corresponding feature vector. Given $h$ and the right feature representation, a search algorithm can compute subjective interestingness scores for each pattern $p \in \mathcal{L}$ and hence use this as optimization criterion.

Devising a generic search algorithm for any type of pattern and ranking function $h$ is an interesting and challenging open research problem on itself. We therefore leave this for future work and only specify an instance tailored for subgroup discovery in the next section.

\section{Learning itemset and subgroup rankings}

We now describe two instances of our proposed approach, for two types of pattern mining: (frequent) itemset mining and subgroup discovery. The key choices concern the source ranking and the pattern features. In making these choices, we aim to keep things as simple as possible, in order to avoid the necessity of data mining expertise and hence making our approach accessible to domain experts as well.

\subsection{Frequent itemset mining}

For itemset mining, in absence of any prior knowledge, the ranking according to frequency is the most natural choice of the source ranking. We consider the following features for itemsets:

- Attribute ${ }_{i}$ : a binary feature for each item; equals 1 iff the corresponding item belongs to the itemset.

- Cover $_{T}$ : a binary feature for each transaction; equals 1 iff the corresponding transaction is covered by the itemset.

- Frequency: a numeric feature; the frequency of the itemset.

- Length: a numeric feature; the size of the itemset, i.e., $|I|$.

The total number of features depends on the dimensions of the data, and is equal to $|\mathcal{I}|+|\mathcal{D}|+2$.

\subsection{Subgroup discovery}

In case of subgroup discovery, there is more information than just frequency that can be used to start from a -potentially- better source ranking. That is, any objective subgroup interestingness measure $\varphi$ can be used, e.g, Sensitivity or Specificity. A standard subgroup discovery algorithm can be used to mine the initial pattern set and corresponding source ranking. 
In addition to the features described for frequent itemset mining, which can be used in almost any pattern mining setting, we consider the following features specific to subgroup discovery:

- Positive Frequency: a numeric feature; the frequency of the positive labels in the subgroup, i.e., $\frac{\left|C^{1}\right|}{\left|\mathcal{D}^{1}\right|}$.

- Negative Frequency: a numeric feature, the frequency of the negative labels in the subgroup, i.e., $\frac{\left|C^{0}\right|}{\left|\mathcal{D}^{0}\right|}$.

- Interestingness: a numeric feature; $\varphi$.

Also, due to a richer pattern language, feature sets Length and Attribute $_{A}$ have a slightly different interpretation in case of subgroup discovery: Length is equal to the number of conditions in the description, and each attribute is still represented by a single binary feature Atribute $_{A}$, even if it occurs in multiple conditions. For example, if $A_{1}$ and $A_{2}$ are numeric attributes, a subgroup $A_{1}>1 \wedge A_{1}<2 \wedge A_{2}>0$ has Length $=3$, Attribute $\left(A_{1}\right)=1$, and Attribute $\left(A_{2}\right)=1$.

In order to use the learned ranking function $h$ for mining new patterns, we employ a beam search-based subgroup discovery algorithm, DSSD ${ }^{27}$. At each level, $h$ is used to rank candidates in the beam; no other changes to the algorithm are necessary.

\section{Experiments}

In previous sections we described a framework for interactive learning of pattern ranking functions. The key research question is: "Is it possible to learn preferences over patterns, given only sample rankings as input?" We demonstrate that the answer is positive and proceed with answering the following more specific research questions:

Q1) Which ranking algorithms are most suitable for this purpose?

Q2) For which pattern types is learning feasible? If so, how much training data is required?

Q3) Which pattern features are important for learning?

Q4) Does active learning reduce the user effort? Which query selection methods perform better with respect to various performance measures?

Q5) Do the learned ranking functions enable the discovery of novel interesting patterns when used as search heuristics?

\subsection{Evaluation methodology}

User feedback emulation Evaluating interactive data mining algorithms is hard, for experts are scarce, and it is virtually impossible to collect enough data for drawing reliable conclusions. In order to perform an extensive evaluation we use 
an objective ranking of patterns as the target ranking. We emulate user feedback by ranking patterns using an objective interestingness measure (target measure), which is not known to the learning algorithm. We use Surprisingness for itemset mining and $\chi^{2}$ for subgroup discovery (see Section 3 for definitions).

Performance measures Given a set of patterns $\mathcal{P}$, the goal of learning rankings is two-fold: 1) to identify subjectively interesting patterns in $\mathcal{P}$ and 2) to learn an accurate overall ranking of $\mathcal{P}$. Therefore, we use several ranking distance measures to quantify learning performance. Let $R_{\mathcal{P}}^{*}$ denote the target ranking of $\mathcal{P}, \hat{R}_{\mathcal{P}}$ the learned ranking, and $\hat{R}_{\mathcal{P}}(i)$ the learned rank of the $i$-th element in the target ranking:

1) In order to evaluate the capacity of the algorithm to identify the most interesting patterns in $\mathcal{P}$, we consider Recall at $k$ :

$$
\operatorname{Rec}_{k}=\left|\left\{i \in\{1,2, \ldots, k\} \mid \hat{R}_{\mathcal{P}}(i) \leq k\right\}\right|
$$

2) In order to evaluate the overall ranking accuracy, we consider rank correlation and discounted error. Spearman's rank correlation coefficient $\rho$ is based on the sum of squared differences between learned and target ranks for each element:

$$
\rho=1-\frac{6 D_{s}\left(R_{\mathcal{P}}^{*}, \hat{R}_{\mathcal{P}}\right)}{|\mathcal{P}|(|\mathcal{P}|-1)}, \text { where } D_{s}=\sum\left(i-\hat{R}_{\mathcal{P}}(i)\right)^{2}
$$

Rank correlation essentially assigns equal weights to all elements, whereas Discounted Error $D E$ assigns larger weights to higher-ranked elements:

$$
D E=\sum \frac{\left|i-\hat{R}_{\mathcal{P}}(i)\right|}{\ln (i+1)}
$$

We use $\rho$ as the primary performance measure in the exploratory experiments.

Performance measures calculated for the entire ranking, such as $\rho$ or $D E$, are less relevant if the ultimate goal is to identify top-ranking patterns. However, if the goal is to learn a search heuristic, the capacity to correctly identify low-ranked patterns is important as well. Note that reported values of $D E$ are normalized to the range of $[0,1]$.

In order to estimate the convergence rate of the algorithm, for each performance measure we report values of the area under performance curve $(A U C)$ in addition to absolute values. The performance curves are constructed as follows: for each iteration $i$, the value of a performance measure after $i$ iterations is recorded. The larger the area, the fewer iterations are required to attain high values of the performance measure.

To quantify user effort, we use the total number of distinct queried pairs $C_{F}$ : $C_{F}=\left|\left\{\left(p_{i k}, p_{j k}\right) \mid f_{k} \in F ; p_{i k}, p_{j k} \in f_{k}\right\}\right| . C_{F}$ is equal to the number of pairwise preferences that a user has to compute in order to provide the feedback. 
Table 6. Datasets and pattern sets used in experiments. For each dataset, source rankings of 1000 patterns were mined using various objective interestingness measures: Frequency for both itemset mining and subgroup discovery, and Sensitivity and Specificity for subgroup discovery. For each source ranking, Spearman's rank correlation $\rho$ between the source ranking and the target ranking is reported.

Setting

Source rankings

\begin{tabular}{|c|c|c|c|c|c|}
\hline \multicolumn{3}{|c|}{ Subgroup discovery (SD) } & Frequency & Sensitivity & Specificity \\
\hline Dataset & $|\mathcal{D}|$ & $|A|$ & \multicolumn{3}{|c|}{$\rho($ Source, Target $)$} \\
\hline breast-w & 683 & 9 & 0.26 & 0.61 & 0.02 \\
\hline credit-a & 653 & 15 & -0.26 & -0.06 & 0.51 \\
\hline credit-g & 1000 & 20 & 0.11 & 0.33 & 0.86 \\
\hline diabetes & 768 & 8 & -0.01 & 0.17 & 0.43 \\
\hline vote & 232 & 16 & 0.33 & 0.84 & 0.51 \\
\hline \multicolumn{3}{|c|}{ Itemset mining (FIM) } & Frequency & & \\
\hline Dataset & $|\mathcal{D}|$ & $|A|$ & \multicolumn{3}{|c|}{$\rho($ Source, Target $)$} \\
\hline anneal & 812 & 94 & -0.31 & & \\
\hline australian-credit & 653 & 125 & -0.27 & & \\
\hline german-credit & 1000 & 112 & -0.23 & & \\
\hline heart-cleveland & 296 & 95 & -0.21 & & \\
\hline hepatitis & 137 & 68 & -0.24 & & \\
\hline lymph & 148 & 68 & 0.03 & & \\
\hline primary-tumor & 336 & 31 & -0.07 & & \\
\hline soybean & 630 & 50 & 0.09 & & \\
\hline tic-tac-toe & 958 & 27 & 0.12 & & \\
\hline vote & 435 & 48 & -0.13 & & \\
\hline zoo-1 & 101 & 36 & -0.18 & & \\
\hline
\end{tabular}

Datasets For our empirical evaluation we used datasets from publicly available repositories: 11 datasets for itemset mining were taken from the CP4IM repository ${ }^{\mathrm{a}}$; 5 datasets for subgroup discovery were taken from the UCI repository ${ }^{\mathrm{b}}$. Tuples with missing attribute values were removed from all datasets.

Source rankings The 1000 most frequent closed itemsets, ranked by their frequencies, were used as source rankings for experiments with itemset mining. Source subgroup rankings were mined using DSSD with the following parameters (see Van Leeuwen and Knobbe ${ }^{27}$ for details): minimal frequency $=0.1|\mathcal{D}|$, beam width $=100$, maximal depth $=5$. Numeric attributes were discretized onthe-fly by local binning of occurring values into 6 equal-sized bins. The cover-based beam selection heuristic was applied with the default trade-off parameter settings. 10000 subgroups were mined initially, then 1000 subgroups were selected from this

\footnotetext{
${ }^{a}$ http://dtai.cs.kuleuven.be/CP4IM/datasets/

${ }^{b}$ http://archive.ics.uci.edu/ml/
} 


$\begin{array}{lrrr}\text { Table } & 7 . \\ \text { SVM pomparison of ranking algorithms. RANKING } \\ \text { erage rank correlation } \rho \text { and has the lowest runtime. } \\ \end{array}$

large set using the same selection heuristic. For each dataset, three subgroup sets were mined using one of the following subgroup interestingness measures, Sensitivity, Specificity, or Frequency (essentially a non-supervised measure).

We have intentionally chosen simple source measures so that source and target rankings are substantially different, and hence the learning problem is challenging. Table 6 presents the characteristics of the datasets and corresponding pattern sets, including the initial rank correlation $\rho_{0}$ between the source ranking and the target ranking. Most source rankings by Frequency are weakly or negatively correlated with the respective target rankings, whereas source rankings by supervised measures Sensitivity and Specificity are better correlated with the target rankings by $\chi^{2}$. In the experiments, we investigate which effect this has on learning performance.

\subsection{Experimental results}

Q1) Comparison of ranking algorithms We first turn to comparing the learning algorithms listed in Section 5: ListNet, RANKBoost, and RANKNET as implemented in the RANKLIB libraryc ${ }^{\mathrm{c}}$; the standard implementation of RANKING SVM ${ }^{\mathrm{d}}$; and our own implementation of SCD.

We use default parameter values in the implementations or values recommended in the original papers: LISTNET(1500 epochs, learning rate $=0.00001$, no hidden layers); RANKBoOsT(300 training rounds, 10 threshold candidates); RANKNET(100 epochs, learning rate $=0.00005,1$ hidden layer with 10 nodes); RANkING SVM(trade-off $C=0.005)$ with a linear kernel, per recommendations of the authors, it is increased after each iteration, i.e. the effective value is $C_{0} \times$ iteration; $\mathrm{SCD}(1000$ iterations, regularization parameter $=0.001)$.

For these experiments, random queries are used as training data. 10 patterns are selected uniformly at random (without replacement) from each source ranking and ranked by the target measure. All algorithms use the same training data. This procedure is repeated 10 times for each source ranking; average values of performance measures are reported. Pattern sets are grouped by the source quality measure, and results are aggregated over all datasets. Results are shown in Table 7.

\footnotetext{
${ }^{c}$ http://sourceforge.net/p/lemur/wiki/RankLib/

d http://www.cs.cornell.edu/people/tj/svm_light/svm_rank.html
} 
Fig. 1. Estimating the required amount of training data. Reasonably small training data of 30 ranked patterns or less suffice to attain high values of rank correlation, $\rho \geq 0.7$, Less training data is required, if the source ranking is better correlated with the target ranking, i.e. for the subgroup discovery task and Sensitivity and Specificity source rankings.

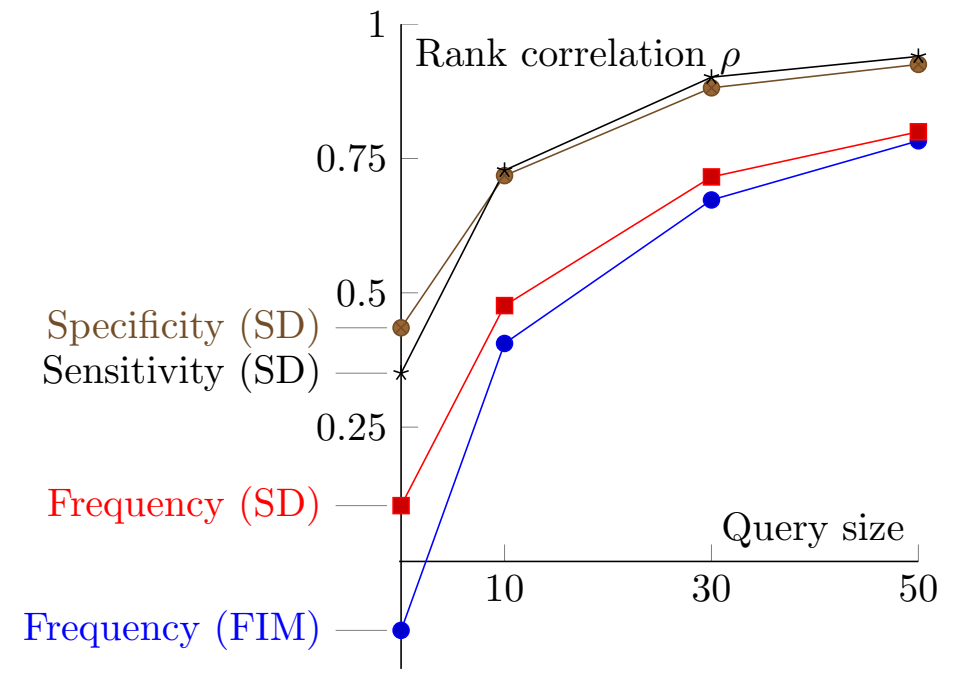

RANKING SVM, SCD, and RANKBoOST are able to learn sufficiently accurate rankings, $\rho \gtrsim 0.4$, which indirectly confirms feasibility of our approach. The only listwise algorithm, LISTNET, does not perform well, neither does the other neural network-based algorithm RANKNET. The results are consistent across pattern types.

We use RANKING SVM in the following experiments, as it provides the highest performance and has the lowest runtime among the evaluated algorithms. Note that on average, one learning iteration takes approximately $0.1 \mathrm{~s}$, therefore in principle, this implementation can be used in a truly interactive setting.

Q2) Estimating the required amount of training data In order to estimate the amount of required training data, we select uniformly at random $S$ patterns from each source ranking and use them as training data. The average rank correlation over 10 experiments is reported. Figure 1 shows the results for $S \in\{0,10,30,50\}$, where $S=0$ corresponds to the correlation between source and target rankings.

The results show that learning accurate ranking functions requires a reasonable amount of training data: querying at most 30 patterns out of 1000 allows attaining high values of ranking correlation, $\rho \geq 0.7$. They also demonstrate the importance of prior beliefs, i.e. the choice of the source ranking: less training data is required, if the source ranking is better correlated with the target ranking, as is the case for $\chi^{2}$ and Sensitivity or Specificity. Furthermore, the results with the Frequency source ranking are very similar for itemsets and subgroups.

Although these results suggest that preference learning is a suitable technique for ranking patterns, querying 30 patterns at once incurs considerable costs, $C_{F}=\left(\begin{array}{c}30 \\ 2\end{array}\right)=435$, which might be prohibitively large for a human user. Later, we demonstrate that active learning helps reduce the required user effort. 
Table 8. Evaluating the importance of pattern features. We construct feature representations of patterns incrementally, i.e. we start with an empty representation and add feature sets one by one, based on the improvement of rank correlation $\rho$ that they enable. Features related to the target measures are considerably more likely to be included in the best feature sets, e.g. Length for Surprisingness, or Pos./Neg.frequency for $\chi 2$. For each source ranking, 10 experiments with randomly generated training data were conducted. The first two columns show the probability of a feature set being added at the first iteration and the average attained value of $\rho$. The rightmost columns show the probability of a feature set being included in the best feature set.

\begin{tabular}{|c|c|c|c|c|c|c|c|}
\hline \multirow{4}{*}{ Feature set } & & & \multirow{4}{*}{$\begin{array}{c}\text { In best } \\
\text { Prob. }\end{array}$} & \multirow{3}{*}{ Feature set } & & & \multirow{3}{*}{$\begin{array}{c}\text { In best } \\
\text { Prob. }\end{array}$} \\
\hline & & & & & \multicolumn{2}{|c|}{ First added } & \\
\hline & \multicolumn{2}{|c|}{ First added } & & & Prob. & $\rho$ & \\
\hline & Prob. & $\rho$ & & Pos.frequency & 0.16 & 0.54 & 0.75 \\
\hline \multirow{5}{*}{$\begin{array}{l}\text { Length } \\
\text { Cover } \\
\text { Attributes } \\
\text { Support }\end{array}$} & \multirow{5}{*}{$\begin{array}{l}0.29 \\
0.55 \\
0.16 \\
0.00\end{array}$} & \multirow{5}{*}{$\begin{array}{l}0.38 \\
0.53 \\
0.38 \\
0.01\end{array}$} & \multirow{5}{*}{$\begin{array}{l}0.90 \\
0.58 \\
0.50 \\
0.30\end{array}$} & Cover & 0.65 & 0.81 & 0.66 \\
\hline & & & & Neg.frequency & 0.15 & 0.58 & 0.65 \\
\hline & & & & Quality & 0.00 & 0.32 & 0.41 \\
\hline & & & & Support & 0.00 & 0.59 & 0.37 \\
\hline & & & & Attributes & 0.03 & 0.47 & 0.29 \\
\hline & Items & inin & & Length & 0.00 & 0.23 & 0.25 \\
\hline
\end{tabular}

(b) Subgroup discovery

Q3) Evaluating the importance of pattern features In order to evaluate the importance of various feature sets we performed the following procedure. Similar to the previous experiments, random subsets of $\mathcal{P}$ are used as the training data. For each selection of training data, we incrementally construct the pattern representation. At each step the feature set that results in the largest increase of $\rho$ is added to the representation. Note that feature sets such as Attribute or Cover are added as a whole, as opposed to adding features for each attribute or tuple individually. The procedure continues as long as $\rho$ increases.

For each pattern type, we consider all feature sets described in Section 6. Note that all numeric features are discretized into 5 bins. The size of the training data is 30 subgroups. For each subgroup set, the training data selection procedure was performed 10 times; average values are reported. Results are shown in Table 8.

The importance of features depends on the pattern type and the target measure. For itemset mining, Length was the most likely to be included in the best feature set, because long itemsets tend to have higher values of Surprisingness. Attributes are important as well, because individual item frequencies are directly included in the formula of Surprisingness. For subgroup discovery, features that are included in the formula of $\chi^{2}$ are likewise important, for example Pos./Neg.frequency. Cover is important in both cases, because this feature set helps capture interactions between other features, albeit indirectly. These results also show that the learned weights are interpretable, i.e. that the algorithm not only learns accurate rankings, but can also provide explanations, which is necessary for human users.

In the remaining experiments, we use the following feature representations: \{Attributes, Cover, Length\} for itemsets mining; and Attributes, Cover, PositiveFrequency, NegativeFrequency $\}$ for subgroup discovery. 
Table 9. Tuning IR-inspired active learning heuristics. For each source ranking, we rank parameter values according to attained values of rank correlation $\rho$ and report average ranks across all source rankings. Lower values of $\alpha$ and $\beta$, i.e., increasing diversity of selected queries, improves the performance of IR-inspired selectors. We include $\operatorname{MMR}(0.3), \operatorname{RDD}(0.15$, $0.15)$, and GlobalmMR(0.3) in our experiments. The effect of the interpolation coefficient $\mu$ is not substantial; we use $\mu=0.5$ in experiments.

\begin{tabular}{lrr}
$\alpha$ & Avg.rank & Avg. $\rho$ \\
\hline 0.3 & 2.1 & 0.52 \\
0.1 & 2.7 & 0.52 \\
0.5 & 3.1 & 0.41 \\
0.9 & 3.4 & 0.37 \\
0.7 & 3.6 & 0.35
\end{tabular}

a) MMR

\begin{tabular}{lrr}
$\alpha$ & Avg.rank & Avg. $\rho$ \\
\hline 0.3 & 1.8 & 0.67 \\
0.1 & 1.9 & 0.68 \\
0.5 & 2.9 & 0.57 \\
0.7 & 3.9 & 0.49 \\
0.9 & 4.5 & 0.42
\end{tabular}

c) GlobalmmR

\begin{tabular}{llrr}
$\alpha$ & $\beta$ & Avg.rank & Avg. $\rho$ \\
\hline 0.15 & 0.15 & 2.6 & 0.54 \\
0.1 & 0.2 & 2.7 & 0.55 \\
0.2 & 0.1 & 2.7 & 0.53 \\
0.25 & 0.25 & 3.1 & 0.47 \\
0.45 & 0.45 & 3.8 & 0.36
\end{tabular}

b) RDD

\begin{tabular}{crr}
$\mu$ & Avg.rank & Avg. $\rho$ \\
\hline 0.5 & 2.3 & 0.58 \\
0.7 & 2.6 & 0.56 \\
0.3 & 2.7 & 0.55 \\
0.9 & 2.9 & 0.51 \\
0.1 & 3.0 & 0.52
\end{tabular}

d) Interpolation

Q4) Query selection We now present the comparison of query selection strategies. We quantify performance by average ranks of strategies with respect to various performance measures. For each source ranking, various query selectors were evaluated and ranked according to AUC for respective performance measures. Tied ranks are assigned the highest rank from the equivalent range. Finally, ranks for a specific query selector are averaged over all pattern sets.

Setting parameters First, we briefly describe how to set parameters of query selectors. For IR-inspired selectors MMR and GLOBALMMR, we first fix the interpolation coefficient $\mu=0.5$ and vary the value of $\alpha$ (Table 9). The larger focus on query diversity (lower values of $\alpha$ ) results in the highest performance; we will use $\alpha=0.3$ in experiments. For RDD, we essentially keep the same weight assigned to the diversity component (0.7) and vary the values of $\alpha$ and $\beta$ so that $\alpha+\beta=0.3$ (for completeness, we also provide results for two combinations with a lower diversity weight). The performance is slightly better than that of MMR and does not differ substantially for various combinations of $\alpha$ and $\beta$; we will use $\alpha=0.15, \beta=0.15$ in experiments. Finally, for the chosen parameter values, we vary the value of $\mu$. The effect on performance is small; we will use $\mu=0.5$ in experiments. Note that we always use the Euclidean distance measure.

For SVMBATCH, we first turn off candidate set pruning and vary the values of the uncertainty weight $\lambda$ (Table 10). In line with original findings ${ }^{5}$, the effect on performance is small; we will use $\lambda=0.3$ in experiments. Then, for the chosen $\lambda$, we experiment with values of the pruning threshold minlen, where minlen $=x$ 
Table 10. Tuning an uncertainty-based active learning heuristic SVMBATCH. Performance of SVMBATCH does not depend substantially on the uncertainty weight $\lambda$. Pruning a candidate set can improve performance. We use $\lambda=0.3$ and minlen $=0.3$ in experiments.

\begin{tabular}{rrr}
\hline$\lambda$ & Avg.rank & Avg. $\rho$ \\
\hline 0.3 & 2.8 & 0.6936 \\
0.7 & 2.8 & 0.6835 \\
0.5 & 3.0 & 0.6614 \\
0.1 & 3.1 & 0.6878 \\
0.9 & 3.2 & 0.6604 \\
\hline
\end{tabular}

\begin{tabular}{rrr}
\hline minlen & Avg.rank & Avg. $\rho$ \\
\hline 0.3 & 1.96 & 0.7050 \\
- & 2.65 & 0.6936 \\
0.1 & 3.08 & 0.6764 \\
\hline
\end{tabular}

denotes pruning all candidate pairs with the norm less than $x \cdot \sqrt{2 d}$ and $\sqrt{2 d}$ is the maximal norm of a binary vector of the dimensionality $d$ (the dimensionality of a pattern feature vector depends on the dimensions of the dataset and the chosen feature sets). We observe that pruning can potentially improve the performance, hence we will use minlen $=0.3$ in experiments. Note that larger values of minlen in certain cases can result in overly eager pruning and hence in empty candidate sets; therefore they are not reported in the table.

Comparison of query selection heuristics Following the results of previous experiments, we compare the following heuristics: IR-inspired selectors $\operatorname{MMR}(\alpha=$ $0.3), \operatorname{RDD}(\alpha=0.1, \beta=0.2)$, and $\operatorname{GlobalmMR}(\alpha=0.3)$ with $\mu=0.5$ and the Euclidean distance measure; $\operatorname{SVMBatch}(\lambda=0.1$, minlen $=0.1)$. A nonbiased randomized strategy RANDOM, which selects subsets of the source ranking uniformly at random, is used as a baseline. To compute the ranks of RANDOM, for each experimental setting, 10 experiments were conducted, and median values of performance measures were used.

All experiments were conducted with 10 iterations and query size $S=5$. The maximal effort is then $C_{F}=10 \times\left(\begin{array}{l}5 \\ 2\end{array}\right)=100$. A single query of 15 patterns has roughly equivalent costs, $C_{F}=\left(\begin{array}{c}15 \\ 2\end{array}\right)=105$, therefore we report the median performance over 10 experiments with RANDOM and $S=15$ as a non-iterative baseline.

Table 11 presents the aggregate results regarding the performance of query selectors. They show that global query diversity is required to learn accurate overall rankings: methods that ensure global diversity, i.e., GlobalMMR, SVMBATCh, and RANDOM, attain the highest values of $\rho$ and $D E$. However, active learning heuristics slightly outperform RANDOM in terms of $D E$, i.e., they are more accurate at the top of the ranking. The performance of IR-inspired selectors, MMR and $\mathrm{RDD}$, is substantially lower, but acceptable, i.e., it is comparable to the baseline. However, they incur considerably lower costs: they query approximately two times fewer pattern pairs. Also, their recall at the top of the ranking is substantially larger than for the random query selection.

Table 12 shows results grouped by source measures. The performance of active learning strongly depends on the source ranking. For the source rankings 
Table 11. Comparison of active learning heuristics. For each source ranking, heuristics are ranked based on the values of respective performance measures; we report the average ranks across all source rankings and average values of performance measures after 10 iterations. Performance of all heuristics is comparable to the non-iterative baseline. Methods that ensure global diversity, GLOBALMMR and SVMBATCH, result in accurate overall rankings, i.e. rank highly according to rank correlation $\rho$ and discounted error DE. MMR and RDD provide slightly lower performance, but at considerably lower costs $C_{F}$. Iterative random query selection performs well in terms of learning overall rankings $(\rho)$, but is outperformed in terms of recall at the top of the ranking ( $\operatorname{Rec}_{10}$ and $\left.R e c_{100}\right)$.

\begin{tabular}{lrrrrrrrrrrr}
\hline & \multicolumn{1}{c}{ Left column: average rank. Right column: average value after } & 10 iterations. \\
Selector & \multicolumn{2}{c}{$\rho$} & \multicolumn{2}{c}{$D E$} & \multicolumn{1}{c}{$R e c_{10}$} & \multicolumn{2}{c}{$R e c_{100}$} & \multicolumn{1}{c}{$C_{F}$} \\
\hline SVMBATCH & 2.2 & 0.73 & 2.2 & 0.24 & 1.5 & 0.4 & 2.2 & 0.67 & 3.3 & 99.9 \\
GLOBALMMR & 2.3 & 0.73 & 2.2 & 0.24 & 1.3 & 0.4 & 2.2 & 0.69 & 3.1 & 94.2 \\
RANDOM & 3 & 0.74 & 3.3 & 0.26 & 3 & 0.2 & 3.8 & 0.58 & 3.2 & 100 \\
RDD & 3.5 & 0.64 & 3.4 & 0.32 & 2.2 & 0.3 & 3.2 & 0.56 & 1.6 & 46.2 \\
MMR & 3.7 & 0.63 & 3.5 & 0.32 & 2.3 & 0.3 & 3.1 & 0.58 & 1.4 & 44.1 \\
\hline RANDOM $(\mathrm{S}=15)$ & & 0.64 & & 0.32 & & 0.1 & & 0.48 & & 105 \\
\hline
\end{tabular}

highly correlated with the target ranking, i.e., the subgroup discovery task and the Sensitivity and Specificity source rankings, active learning heuristics outperform random query selection according to most performance measures.

Q5) Generalizing to the entire pattern language Finally, we evaluate the capacity of learned ranking functions to generalize to unobserved patterns: we estimate the target interestingness of top- $k$ patterns according to learned ranking functions, which do not necessarily belong to the source ranking $\mathcal{P}$, and compare it with the interestingness of patterns that are obtained by the search guided by the target measures directly. We use $k=1000$.

For itemset mining, we first mine a complete collection of frequent itemsets at $\sigma=0.1$ and rank it using Surprisingness and the learned ranking function $h$ to obtain the top- $k$ patterns. We restrict ourselves to the datasets that contain less than 1 million itemsets at this support threshold: primary-tumor (50040 itemsets), soybean (27635 itemsets), tic-tac-toe (1661 itemsets), vote (49097 itemsets), and zoo-1 (151806 itemsets).

For subgroup discovery, we use DSSD to search with $\chi^{2}$ and its extension as described in Section 6 to search with the learned ranking functions. Search parameters were identical to the parameters used for mining the source rankings, and learning parameters were identical to the ones used in the query selection experiments.

The results confirm the generalization capacity of learned ranking functions (Table 13): median values of the target measures of top $k$ patterns according to $h$ increase substantially, when compared to source rankings. Maximal values are comparable to what can be achieved with direct search. Moreover, learning accurate rankings increases the magnitude of improvement. For this reason, GLOBALMMR or SVMBATCH result in better generalization than MMR. 
Table 12. Comparison of active learning heuristics; results are grouped by source measures. For each source ranking, heuristics are ranked based on the values of respective performance measures; we report the average ranks across all source rankings and average values of performance measures after 10 iterations. Performance of query selectors depends on the source ranking. Active learning outperforms iterative random query selection both with respect to overall ranking correlation $\rho$ and recall at the top of the ranking $\operatorname{Rec}_{10}$, when the source ranking is correlated with the target ranking, i.e. for the subgroup discovery setting and the Sensitivity or Specificity source rankings.

\begin{tabular}{|c|c|c|c|c|c|c|}
\hline Task & $\begin{array}{l}\text { Left colum } \\
\text { Source }\end{array}$ & $\begin{array}{l}\text { rank. Right column } \\
\text { Selector }\end{array}$ & \multicolumn{2}{|c|}{$\rho$} & \multicolumn{2}{|c|}{$R e c_{10}$} \\
\hline \multirow{15}{*}{ Subgroup discovery } & \multirow{5}{*}{ Frequency } & RANDOM & 1.5 & 0.71 & 3.8 & 0.2 \\
\hline & & GLOBALMMR & 2.2 & 0.59 & 1.4 & 0.6 \\
\hline & & SVMBATCH & 2.8 & 0.46 & 2.4 & 0.5 \\
\hline & & MMR & 4 & 0.38 & 3.2 & 0.3 \\
\hline & & RDD & 4.4 & 0.4 & 3.2 & 0.2 \\
\hline & & SVMBATCH & 1.2 & 0.94 & 1.6 & 0.8 \\
\hline & & GLOBALMMR & 2 & 0.95 & 2 & 0.8 \\
\hline & Sensitivity & $\mathrm{RDD}$ & 3.6 & 0.85 & 2.8 & 0.6 \\
\hline & & MMR & 3.6 & 0.89 & 3.4 & 0.6 \\
\hline & & RANDOM & 4 & 0.87 & 4.3 & 0.2 \\
\hline & & SVMBATCH & 1.2 & 0.93 & 1.8 & 0.8 \\
\hline & & GLOBALMMR & 2.2 & 0.91 & 1.4 & 0.9 \\
\hline & Specificity & $\mathrm{RDD}$ & 3.4 & 0.86 & 3.2 & 0.7 \\
\hline & & RANDOM & 3.7 & 0.85 & 4.3 & 0.5 \\
\hline & & MMR & 4 & 0.86 & 3 & 0.7 \\
\hline \multirow{5}{*}{ Itemset mining } & \multirow{5}{*}{ Frequency } & GLOBALMMR & 2.5 & 0.62 & 2.5 & 0.2 \\
\hline & & SVMBATCH & 2.9 & 0.66 & 2.9 & 0.2 \\
\hline & & RANDOM & 3 & 0.62 & 3 & 0.1 \\
\hline & & RDD & 3.2 & 0.55 & 3.2 & 0.1 \\
\hline & & MMR & 3.5 & 0.52 & 3.5 & 0.1 \\
\hline
\end{tabular}

The generalization performance is lower in the case of itemset mining, due to a source ranking that is less correlated with the target. This makes overfitting more likely; in other words, the learned ranking functions are only applicable to $\mathcal{P}$, but not to the entire $\mathcal{L}$. This is the case for SVMBATCH: larger values of $\rho$ result in lower Surprisingness of top-ranked itemsets.

Figure 2 presents a detailed view of two experiments with SVMBATCH, with the dataset primary tumor for itemset mining and the dataset credit- $a$ and the source ranking by Specificity. The ranking functions learned after 1, 2, 5, and 10 iterations were used in the search. The boxplots show the distribution of the target measures (Surprisingness and $\chi^{2}$ respectively) in the set of top-1000 patterns according to the learned ranking function. They illustrate the phenomena discussed in the previous paragraph. For itemset mining, overfitting results in decrease of the maximal Surprisingness after more learning iterations. Nevertheless, the median gradually increases. For subgroup discovery, the more learning iterations are performed, the more the distributions are skewed towards high values of $\chi^{2}$. Median and maximal values are comparable to ones obtained with $\chi^{2}$ used directly as a search heuristic. 
Table 13. Evaluating generalization capacity of learned ranking functions. For each dataset, we learn a ranking function $h$ for a number of iterations and use it to mine novel subgroups or rank a complete collection of frequent itemsets. $\Delta \varphi_{\text {med }}$ (resp. $\Delta \varphi_{\text {med }}^{*}$ ) denote the ratio between the median values of the target measure $\varphi$ in top 1000 patterns according to $h$ (mined or ranked) and in the source ranking (resp. in top 1000 subgroups according to $\varphi$ directly), whereas $\Delta \varphi_{\max }$ and $\Delta \varphi_{\max }^{*}$ denote the ratios between the maximal values of $\varphi$ in respective sets. For each selector, we report the median values of these ratios across all datasets and source rankings. Learned ranking functions generalize beyond the source rankings, as evidenced by the increase of median target measure values $\left(\Delta \varphi_{\text {med }}\right)$. Learning accurate overall rankings (higher values of $\rho$ ) improves quality of discovered patterns, although the effect is more pronounced for subgroup discovery than for itemset mining.

\begin{tabular}{|c|c|c|c|c|c|c|c|}
\hline Setting & Selector & Iterations & Avg. $\rho$ & $\Delta \varphi_{\text {med }}$ & $\Delta \varphi_{\max }$ & $\Delta \varphi_{\text {med }}^{*}$ & $\Delta \varphi_{\max }^{*}$ \\
\hline \multirow{6}{*}{$\mathrm{SD}$} & \multirow{2}{*}{ GlobalMMR } & 5 & 0.6574 & 1.85 & 1.03 & 0.54 & 0.96 \\
\hline & & 10 & 0.7443 & 2.05 & 1.03 & 0.64 & 0.96 \\
\hline & \multirow{2}{*}{ MMR } & 5 & 0.5952 & 1.73 & 1.02 & 0.34 & 0.94 \\
\hline & & 10 & 0.5845 & 1.7 & 1.01 & 0.27 & 0.92 \\
\hline & \multirow{2}{*}{ SVMBatch } & 5 & 0.6480 & 2.63 & 1.02 & 0.43 & 0.98 \\
\hline & & 10 & 0.7567 & 3.29 & 1.02 & 0.68 & 0.96 \\
\hline \multirow{6}{*}{ FIM } & \multirow{2}{*}{ GlobalMMR } & 5 & 0.4688 & 2.09 & 0.835 & 0.565 & 0.83 \\
\hline & & 10 & 0.6062 & 2.16 & 0.905 & 0.575 & 0.89 \\
\hline & \multirow{2}{*}{ MMR } & 5 & 0.3494 & 2.215 & 0.87 & 0.655 & 0.835 \\
\hline & & 10 & 0.3808 & 2.275 & 0.87 & 0.575 & 0.835 \\
\hline & \multirow{2}{*}{ SVMBatch } & 5 & 0.4143 & 3.38 & 0.925 & 0.68 & 0.89 \\
\hline & & 10 & 0.6318 & 2.735 & 0.84 & 0.54 & 0.805 \\
\hline
\end{tabular}

Fig. 2. Generalization capacity of learned ranking functions. We use a ranking function learned after a certain number of iterations to mine or rank complete collections of patterns. The more learning iterations are performed, the higher the values of the target measure of the patterns discovered with the learned ranking function as a search heuristic. For subgroup discovery, the results after 10 learning iterations are comparable to the search directly guided by the target measure $\chi^{2}$. For itemset mining, the learning is more prone to overfitting, therefore the maximal target interestingness of discovered itemsets tends to decrease. Nevertheless, the median target interestingness gradually increases.

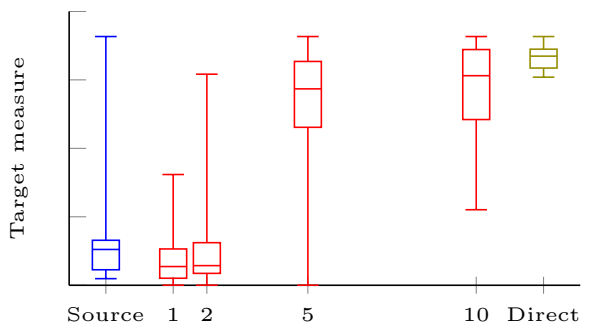

(a) Subgroup discovery:

credit-a, source ranking by Specificity

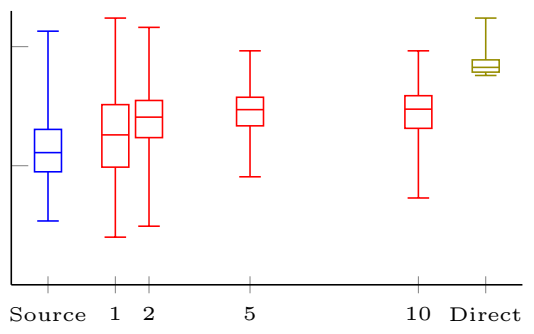

(b) Itemset mining: primary-tumor

\section{Discussion}

We introduced a generic algorithm for the interactive learning of pattern rankings, based on off-the-shelf preference learning techniques and active learning heuristics 
adapted from information retrieval and classification. Furthermore, we presented two instances of this algorithm for well-known pattern mining settings, namely subgroup discovery and itemset mining. Design choices that are specific to each setting include the feature representation of patterns and the choice of source rankings, which represent the prior beliefs of a user. We investigated straightforward and simple options for both of these, by using basic features that follow directly from the problem statement and standard objective measures to define source rankings. Nevertheless, experiments confirm that the proposed algorithm has the capacity to learn accurate pattern rankings in both settings. Moreover, the learned ranking functions generalize beyond the source rankings and hence can be used to mine novel patterns.

These results imply that active preference learning can become an important building block for interactive pattern mining systems, which allow a user to directly influence the mining process so that the results are more relevant to her interests and goals. Such systems should be transparent to non-data mining experts and be able to learn from easy-to-provide feedback. To this end, requiring strict total orders as feedback on complete queries is relatively complicated. Binary feedback, e.g., liking or disliking patterns, is more intuitive for users. In fact, pairwise ranking algorithms, such as RANKING SVM, do not require total orders as input and are directly applicable to any feedback format that can be converted to pairwise preferences. Therefore, designing simpler feedback formats, e.g., implicit feedback that is inferred from user actions, and investigating the effects of coarse-grained feedback on the performance are important future directions.

Source rankings were shown to have a considerable effect on the performance of the learning algorithm. Although this is to be expected, this also introduces a non-trivial parameter for non-expert users. Moreover, if a source ranking does not contain information relevant to the target preferences, the learning algorithm is more prone to overfitting and learned ranking functions do not generalize to the entire pattern language $\mathcal{L}$. One way to alleviate this issue is to move from query selection to query synthesis, i.e., mining novel patterns for querying instead of selecting them from a pre-mined pool. This would produce more representative queries and takes elicited preferences into account more rapidly. Pattern sampling ${ }^{3}$ can be used to achieve these goals without the overhead of exhaustive mining in each iteration.

Finally, to evaluate our algorithm, we emulated the subjective rankings with rankings according to a (latent) objective interestingness measure. These target rankings are total orders, therefore they belong to the hypothesis space $\mathcal{H}$. Whether this assumption holds in practice, i.e., whether genuine subjective pattern rankings can be modeled with total orders, is an open question. Real-world case studies are required to validate the proposed algorithm and this assumption. 


\section{Conclusions}

We presented a general framework for interactive learning of pattern rankings. It requires a user to rank sets of patterns by perceived interestingness and uses preference learning to infer a general ranking function from these sample rankings. An active learning component is used to minimize user effort. The learned ranking functions generalize well and can be used as a search heuristic, enabling the discovery of novel, potentially more interesting patterns.

We applied this framework to two types of pattern mining: frequent itemset mining and subgroup discovery, which can be considered examples of unsupervised and supervised pattern mining respectively. Using a well-principled evaluation method based on user emulation, we demonstrated that it is possible to learn complex preferences over sets of patterns using off-the-shelf preference learning algorithms. Experiments with active learning heuristics showed a trade-off between accuracy of learned rankings and user effort.

Directions for future work include investigating the effect of coarse-grained or noisy feedback on learning performance and shifting from the pool-based active learning to query synthesis, i.e., directly mining patterns for queries. A user study is required to evaluate the practical applicability of the proposed framework.

Acknowledgments This work was supported by the Research FoundationFlanders by means of two Postdoc grants and the project "Instant Interactive Data Exploration" and by the European Commission under the project "Inductive Constraint Programming", contract number FP7-284715.

\section{References}

1. Rakesh Agrawal, Heikki Mannila, Ramakrishnan Srikant, Hannu Toivonen, and A. Inkeri Verkamo. Advances in Knowledge Discovery and Data Mining, chapter Fast Discovery of Association Rules, pages 307-328. 1996.

2. Nir Ailon. An active learning algorithm for ranking from pairwise preferences with an almost optimal query complexity. Journal of Machine Learning Research, 13:137-164, 2012.

3. Mansurul Bhuiyan, Snehasis Mukhopadhyay, and Mohammad Al Hasan. Interactive Pattern Mining on Hidden Data: A Sampling-based Solution. In Proceedings of CIKM, pages 95-104, 2012.

4. Mario Boley, Michael Mampaey, Bo Kang, Pavel Tokmakov, and Stefan Wrobel. One Click Mining - Interactive Local Pattern Discovery through Implicit Preference and Performance Learning. In Interactive Data Exploration and Analytics Workshop at $K D D$, pages 28-36, 2013.

5. Klaus Brinker. Incorporating diversity in active learning with support vector machines. In Proceedings of ICML, pages 59-66, 2003.

6. Chris Burges, Tal Shaked, Erin Renshaw, Ari Lazier, Matt Deeds, Nicole Hamilton, and Greg Hullender. Learning to rank using gradient descent. In Proceedings of ICML, pages 89-96, 2005.

7. Zhe Cao, Tao Qin, Tie-Yan Liu, Ming-Feng Tsai, and Hang Li. Learning to rank: from pairwise approach to listwise approach. In Proceedings of ICML, pages 129-136, 2007. 
8. Tijl De Bie. An Information Theoretic Framework for Data Mining. In Proceedings of $K D D$, pages $564-572,2011$.

9. Vladimir Dzyuba and Matthijs van Leeuwen. Interactive Discovery of Interesting Subgroup Sets. In Proceedings of IDA, pages 150-161, 2013.

10. Vladimir Dzyuba, Matthijs van Leeuwen, Siegfried Nijssen, and Luc De Raedt. Active preference learning for ranking patterns. In Proceedings of ICTAI, pages 532-539, 2013.

11. Yoav Freund, Raj Iyer, Robert E. Schapire, and Yoram Singer. An efficient boosting algorithm for combining preferences. J. Mach. Learn. Res., 4:933-969, December 2003.

12. Eyke Hüllermeier and Johannes Fürnkranz, editors. Preference learning. Springer Berlin Heidelberg, 2011.

13. Szymon Jaroszewicz and Dan A. Simovici. Interestingness of frequent itemsets using Bayesian networks as background knowledge. In Proceedings of KDD, pages 178-186, 2004.

14. Thorsten Joachims. Optimizing search engines using clickthrough data. In Proceedings of KDD, pages 133-142, 2002.

15. Toshihiro Kamishima, Hideto Kazawa, and Shotaro Akaho. A Survey and Empirical Comparison of Object Ranking Methods. In Johannes Fürnkranz and Eyke Hüllermeier, editors, Preference Learning, chapter III, pages 181-202. Springer Berlin Heidelberg, 2011.

16. Willi Klösgen. Advances in Knowledge Discovery and Data Mining, chapter Explora: A Multipattern and Multistrategy Discovery Assistant, pages 249-271. 1996.

17. Petra Kralj Novak, Nada Lavrač, and Geoffrey I. Webb. Supervised descriptive rule discovery: A unifying survey of contrast set, emerging pattern and subgroup mining. Journal of Machine Learning Research, 10:377-403, 2009.

18. Heikki Mannila and Hannu Toivonen. Multiple uses of frequent sets and condensed representations. In Proceedings of the KDD, pages 189-194, 1996.

19. Benjamin Negrevergne, Anton Dries, Tias Guns, and Siegfried Nijssen. Dominance programming for itemset mining. In Proceedings of ICDM, pages 557-566, 2013.

20. Buyue Qian, Xiang Wang, Jun Wang, Hongfei Li, Nan Cao, Weifeng Zhi, and Ian Davidson. Fast Pairwise Query Selection for Large-Scale Active Learning to Rank. In Proceedings of ICDM, pages 607-616, 2013.

21. Filip Radlinski and Thorsten Joachims. Active exploration for learning rankings from clickthrough data. In Proceedings of KDD, pages 570-579, 2007.

22. Stefan Rueping. Ranking interesting subgroups. In Proceedings of ICML, pages 913920, 2009.

23. Shai Shalev-Shwartz and Ambuj Tewari. Stochastic methods for 1 1-regularized loss minimization. The Journal of Machine Learning Research, 12:1865-1892, 2011.

24. Xuehua Shen and ChengXiang Zhai. Active feedback in ad hoc information retrieval. In Proceedings of SIGIR, pages 59-66, 2005.

25. Alexander Tuzhilin. On subjective measures of interestingness in knowledge discovery. In Proceedings of KDD, pages 275-281, 1995.

26. Matthijs van Leeuwen. Interactive data exploration using pattern mining. In Andreas Holzinger and Igor Jurisica, editors, Interactive Knowledge Discovery and Data Mining in Biomedical Informatics, volume 8401 of Lecture Notes in Computer Science, pages 169-182. Springer Berlin Heidelberg, 2014.

27. Matthijs van Leeuwen and Arno Knobbe. Diverse subgroup set discovery. Data Mining and Knowledge Discovery, 25(2):208-242, June 2012.

28. Matthijs van Leeuwen and Jilles Vreeken. Mining and using sets of patterns through compression. In Charu C. Aggarwal and Jiawei Han, editors, Frequent Pattern Mining, 
pages 165-198. Springer International Publishing, 2014.

29. Stefan Wrobel. An algorithm for multi-relational discovery of subgroups. In Proceedings of PKDD, pages 78-87. Springer, Heidelberg, 1997.

30. Dong Xin, Xuehua Shen, Qiaozhu Mei, and Jiawei Han. Discovering Interesting Patterns Through Users Interactive Feedback. In Proceedings of KDD, pages 773-778, 2006.

31. Zhao Xu, Kristian Kersting, and Thorsten Joachims. Fast Active Exploration for Link-Based Preference Learning Using Gaussian Processes. In Proceedings of $E C M L / P K D D$, pages 499-514, 2010.

32. Zuobing $\mathrm{Xu}$, Ram Akella, and Yi Zhang. Incorporating Diversity and Density in Active Learning for Relevance Feedback. In Proceedings of ECIR, pages 246-257, 2007.

33. Albrecht Zimmermann and Siegfried Nijssen. Supervised pattern mining and applications to classification. In Charu C. Aggarwal and Jiawei Han, editors, Frequent Pattern Mining, pages 425-442. Springer International Publishing, 2014. 